\title{
Pratiques de la représentation et sociabilité à Santa
} Cruz de Tenerife

Le cas de René Chassériau, consul de France (1874-1889)

\section{Nathalie Le Brun}

\section{OpenEdition}

\section{Journals}

Édition électronique

URL : https://journals.openedition.org/cher/586

DOI : $10.4000 /$ cher.586

ISSN : 2803-5992

\section{Éditeur}

Presses universitaires de Strasbourg

\section{Édition imprimée}

Date de publication : 10 juin 2021

Pagination : 223-240

ISBN : 9791034400928

ISSN : 1968-035X

\section{Référence électronique}

Nathalie Le Brun, «Pratiques de la représentation et sociabilité à Santa Cruz de Tenerife », reCHERches [En ligne], 26 | 2021, mis en ligne le 15 septembre 2021, consulté le 22 novembre 2021. URL : http:// journals.openedition.org/cher/586 ; DOI : https://doi.org/10.4000/cher.586 


\section{Pratiques de la représentation et sociabilité à Santa Cruz de Tenerife Le cas de René Chassériau, consul de France (1874-1889)}

Nathalie Le Brun*

$\mathrm{E}$ ntre 1870 et 1890, Santa Cruz de Tenerife a été, en sa qualité de capitale des îles Canaries, non seulement le siège des autorités provinciales, mais aussi le lieu de résidence officielle d'une grande partie des consulats étrangers dans l'archipel. Dotée d'un port déclaré d'intérêt général en 1880, cette ville, dont la population approchait les dix-neuf mille habitants en 1887 (Diario de Tenerife 10/12/1889: 2), était un important carrefour maritime pour les échanges intercontinentaux et un observatoire de premier ordre pour s'enquérir de l'avancée des projets européens en Afrique. On voyait régulièrement y faire escale des navires de guerre espagnols ou étrangers transportant officiers haut gradés, administrateurs coloniaux, scientifiques et explorateurs à destination des côtes voisines de l'Afrique, des Caraïbes, de l'Amérique du Sud et même d'Asie.

Cette étude propose une approche politique et culturelle de la représentation consulaire à Santa Cruz de Tenerife à partir des pratiques de sociabilité de René Chassériau (1829?-1904), qui y fut consul de France entre 1874 et 1889. $\mathrm{Au}$ cours de cette période, cruciale du point de vue de la transformation des relations de l'Europe avec le reste du monde, cet ancien sous-préfet et percepteur, ayant préalablement servi la France à Bologne et Cagliari, se trouva dans un premier temps à la tête d'un vice-consulat qui dépendait du consulat de France en Andalousie. En 1880, avec la transformation du poste en consulat, dont la juridiction s'étendait sur toutes les îles de l'archipel canarien, et dont la résidence officielle était la capitale provinciale, les fonctions du consul, ainsi que les pratiques de sociabilité inhérentes à ces dernières, furent accrues. La même année, son grade dans le corps des consuls passa de la seconde classe à la première classe (décret du 20 juillet 1880), avant qu'une promotion l'élève

* Nathalie Le Brun, Université de Strasbourg, UR 4376-CHER, ORCID: 0000-00030708-2064, <nlebrun@unistra.fr>. 
à celui de consul général le 22 octobre $1888^{1}$. C'est donc plus spécifiquement sur la période qui va de 1880 à 1889 , à l'époque où René Chassériau dirigeait le consulat de France aux îles Canaries, que se centrera notre étude. Pendant cette période, le nombre de pays représentés dans la capitale canarienne oscilla entre quinze et vingt, mais le corps consulaire n'était pas homogène. En 1881, il y avait à Santa Cruz de Tenerife douze consuls en exercice pour seize états représentés (Poggi 1881: 290-291). Six ans plus tard, en 1887, le nombre de pays disposant d'une représentation s'élevait à dix-neuf, et le nombre de consuls à quinze, dont un consul général (GDE 1887: 45-85). Toutefois, beaucoup de ces consuls étaient des négociants de nationalité espagnole. La France, la Belgique et la Grande Bretagne firent partie des rares nations à nommer des agents sans liens préalables avec les îles Canaries.

Des travaux récents commencent à remettre en cause la dichotomie courante selon laquelle, au XIX ${ }^{\mathrm{e}}$ siècle, le champ du politique aurait été réservé aux diplomates, et le domaine du commerce, aux consuls (De Goey 2016). La situation géostratégique de certains postes consulaires, éloignés des capitales et situés au cœur de régions où les enjeux militaires et politiques étaient importants - comme c'était le cas des îles Canaries au moment de la colonisation de l'Afrique -, aurait amené les consuls à exercer des fonctions politiques et, parfois, en tant que seuls représentants des États étrangers sur place, à mettre en scène le prestige étatique. Par ailleurs, la représentation consulaire doit être considérée dans sa double dimension, à la fois juridico-politique, car le consul agissait et parlait au nom du pays qui l'avait mandaté tout en incarnant ce dernier (Abélès 1991: 245-246; Sintomer 2013), et symbolique, quand se mettait en place tout l'apparat nécessaire à la figuration publique, ce qui a été défini comme une présentification d'un absent ou l'exhibition d'une présence donnant lieu à une "théâtralisation de la vie sociale» (Chartier 1989: 15141515; Sintomer 2013: 16). À première vue, ces définitions de la représentation ne laissent pas apparaître de convergences avec la notion de sociabilité, même entendue dans son sens le plus large, c'est-à-dire comme un ensemble de rapports volontaires ou imposés, familiaux ou professionnels, actifs ou passifs, qu’un individu entretient avec autrui (Leuwers 2005: $\$ 4$ ). Pourtant, échanges et rencontres sous de multiples formes font bien partie, encore aujourd'hui, des pratiques auxquelles tout chef de mission à l'étranger doit se soumettre afin de remplir pleinement son rôle (Kingston 1998: 85-87).

Les rapports que les consuls de France entretenaient dans un cadre officiel avec les autorités de leur lieu de résidence, le corps consulaire et les officiers généraux de la Marine française étaient régis par un ensemble d'usages locaux et de règles à observer dans les relations internationales, lesquelles leur étaient constamment rappelées par des circulaires ministérielles et des manuels prévus à

1 AMAE, 393QO/890, dépêches ministérielles à R.Chassériau (minutes), Paris, 26/07/1880 et 31/10/1888; dépêche de R. Chassériau au ministre des Affaires étrangères, Tenerife, $18 / 11 / 1888$. 
cet effet, tel le Guide pratique des consulats, un ouvrage qui ne connut pas moins de cinq éditions au cours de la seconde moitié du XIX ${ }^{\mathrm{e}}$ siècle ${ }^{2}$. La sociabilité sera comprise ici comme l'ensemble des relations que René Chassériau fut amené à entretenir avec les autorités territoriales, ses homologues consuls, les notabilités locales, ainsi qu'avec des officiers, des scientifiques et d'autres personnalités qui séjournèrent dans le port de Santa Cruz de Tenerife, aussi bien dans un environnement spatial restreint au consulat que dans celui, plus large, de la ville.

Tout en examinant la place que le consulat de France, en tant qu'entité symbolique représentatrice d'un État étranger, occupait dans la ville par rapport aux institutions, aux corporations et aux sociétés liées au pouvoir, mais aussi celle qu'il occupait en tant que demeure familiale, celle de la famille Chassériau, nous tenterons de mettre en évidence les réseaux qui, grâce à des pratiques de sociabilité, se tissaient entre les membres de la société locale. Cela nous amènera à nous demander dans quelle mesure les consuls, bien qu'enfermés dans une sociabilité fortement ritualisée, pouvaient se détacher du rôle de représentation qui leur était assigné et affirmer leur individualité. Est-il possible d'établir des limites entre l'homme public et l'homme privé? Entre la relation formelle et la relation personnelle? Deux types de sources nous permettront de contextualiser les pratiques de la représentation et de la sociabilité, et de tenter de répondre à ces questions : tout d'abord, la presse de Santa Cruz de Tenerife, dont les directeurs, comme nous le verrons, prenaient part à ces échanges et, dans un second temps, s'en faisaient l'écho auprès de la population par le biais des journaux; ensuite, la correspondance des consuls de France, écrite par eux-mêmes ou sous la dictée, par leurs secrétaires, et adressée aux ministres des Affaires étrangères en poste à Paris.

\section{Le temps de la représentation: du respect du protocole à une sociabilité des élites}

La mise en scène consulaire était tributaire d'un protocole qui fixait non pas des dates, mais des moments où les consuls étaient tenus d'assumer leur rôle de représentant d'un État étranger. Les instructions ministérielles communiquées depuis Paris à leurs agents en distinguaient deux: les visites à l'autorité, ainsi que les fêtes locales et étrangères, dont les françaises (Clercq et Vallat 1858:120123). Toutefois, bien que ces instructions n'aient considéré que les rapports à entretenir avec les autorités territoriales et les autres agents étrangers (Clercq et Vallat 1858: 113), une lecture en corrélation de la correspondance consulaire française et de la presse canarienne fait apparaître que les entités présentes lors des cérémonies officielles qui se déroulaient à Santa Cruz de Tenerife, et auxquelles assistait René Chassériau en tant que consul de France, étaient plus nombreuses. Ces entités variaient selon les circonstances, mais un certain

2 Ouvrage initialement sous la direction d'Alexandre de Clercq et Charles de Vallat, puis de Jules de Clercq à partir de 1898. 
nombre d'entre elles étaient présentes de façon permanente. Il s'agissait, en plus des autorités territoriales (militaires, civiles, municipales) et du corps consulaire, des sociétés patriotiques, culturelles et de bienfaisance, des cercles, ainsi que de la presse ${ }^{3}$. Nous analyserons ici trois types d'occasions au cours desquelles représentation consulaire et sociabilité se combinaientà des degrés différents: les visites rendues à l'autorité; certains événements ponctuels à caractère festif; ainsi que les fêtes et les cérémonies régulières. Nous exclurons les occasions accidentelles telles que les funérailles ou les exhumations, ainsi que les dîners et les loisirs, qui feront l'objet d'une étude séparée.

\section{Visites officielles et visites de courtoisie}

Les visites aux autorités supérieures faisaient partie du protocole auquel devaient se plier les représentants d'un État, non seulement à leur arrivée en terre étrangère, mais aussi de manière régulière, afin de s'acquitter de leurs devoirs de courtoisie auprès de certaines d'entre elles (Calvo 1885: 97). Elles se produisaient également ponctuellement, en des circonstances particulières, par exemple quand des officiers étrangers de haut rang séjournaient dans le port, ou encore au décès d'une personnalité importante, pour présenter des condoléances, comme eut à le faire René Chassériau à la mort du roi Alphonse XII en novembre 18854. Contrairement à d'autres types de rencontres et d'échanges, ces cérémonies ritualisées laissaient peu de place à l'expression des individualités.

C'est avec le capitaine général et le gouverneur civil que les représentants des États étrangers avaient le plus de rapports dans un contexte protocolaire, notamment pour leur prise de fonctions officielle. Le règlement d'admission des consuls étrangers en Espagne de 1848 prévoyait en effet que le capitaine général et le gouverneur civil renseignent l'État sur l'aptitude légale des candidats à exercer leurs fonctions dans le royaume (art. 7) (Castro 1886a: 860), avant que ceux-ci reçoivent, soit directement du roi, soit indirectement, par l'intermédiaire du capitaine général, l'exequatur ou l'autorisation officielle (art. 10) (Castro 1886a: 860). Ce sont également ces mêmes figures de l'autorité qui garantissaient

3 D'autres collectivités pouvaient s'ajouter encore, suivant la nature et l'importance des événements, comme les officiers espagnols et étrangers de passage dans le port, les magistrats, les représentants du Commerce, l'Église, les communautés étrangères, des fonctionnaires de différents corps et grades, des ingénieurs, etc.

4 AMAE, 335CCC/14: R. Chassériau au ministre des Affaires étrangères, Tenerife, 8/12/1885, fol. 44r-48v. Dans la mesure où les documents des archives du ministère des Affaires étrangères qui sont extraits de la série "correspondance consulaire et commerciale» relative au poste consulaire de Santa Cruz de Tenerife (abrégée 335CCC dans les notes) sont des dépêches de René Chassériau adressées depuis Tenerife aux ministres dudit département, nous nous limiterons désormais à indiquer la date de rédaction de ces dernières. 
aux consuls et aux ressortissants de la nation que ceux-ci représentaient la protection de leurs intérêts et le bon déroulement de leurs missions ${ }^{5}$.

La correspondance consulaire et la presse n'ont gardé aucune trace de ce qui correspondrait à un rite d'institution marquant la prise de fonction (Bourdieu apud Abélès 1991: 241) de René Chassériau, mais elles en conservent sur les escales des bâtiments d'État français et le séjour d'officiers généraux dans le port de Santa Cruz de Tenerife. Hormis les visites aux autorités qui avaient lieu pour la prise de fonction officielle des consuls, que ceux-ci faisaient en uniforme et accompagnés du personnel du consulat (Clercq et Vallat 1858: 121; Clercq 1898: 134), les visites de courtoisie au capitaine général que rendaient les personnalités importantes et les officiers supérieurs de passage dans le port s'effectuaient à chaque fois en compagnie du consul ou du vice-consul de leur nation d'origine (Clercq et Vallat 1858: 121). C'était une mission de médiation politique et interculturelle que remplissaient les consuls à ces occasions. Nombreux étaient les bâtiments d'État et les officiers généraux français qui faisaient escale dans la rade de Santa Cruz de Tenerife, ce qui obligeait à respecter un cérémonial naval complexe et sollicitait la présence permanente des consuls dans le port. On pourra citer à cet égard les échanges avec l'autorité qui eurent lieu du 13 au 15 octobre 1874, durant la brève escale de La Gallissonnière, un navire de l'État français portant le pavillon de l'amiral Périgot, commandant en chef de la station navale du Pacifique, lequel se rendait à Dakar pour relever l'amiral Roussin dans le commandement de la flotte française dans les mers du Sud. À cette occasion, l'amiral et son état-major rendirent visite à l'autorité militaire, laquelle s'était empressée "de lui rendre tous les honneurs dus à son rang ${ }^{6}$. Ces honneurs que l'on rendait en Espagne aux amiraux étrangers se déroulaient selon un cérémonial identique à celui qui était appliqué pour l'Armada (Castro 1886b : 273) : salut au canon et à la voix à l'arrivée, port de l'uniforme obligatoire, visite à l'autorité, que celle-ci devait rendre, en principe, dans les vingt-quatre heures.

Comme René Chassériau le fit remarquer quelques années plus tard, lors du stationnement des bâtiments de guerre dans la rade de Santa Cruz, saluts et visites étaient rendus par l'autorité locale «avec toute la correction possible » 7 . En cas d'absence du capitaine général, celui-ci pouvait être remplacé par le général gouverneur militaire, et si l'officier étranger avait à se déplacer pour rencontrer le capitaine général en personne, alors le consul l'accompagnait dans cette démarche. Ce fut le cas, en septembre 1886, quand le contre-amiral Ribell, commandant de la division navale de l'Atlantique sud, qui devait s'entretenir avec le capitaine général Buenaventura Carbó Aloy au sujet de l’installation des

5 Avec la révision du règlement en 1887 , les prérogatives des gouverneurs civils des provinces péninsulaires et des Canaries furent accrues, car désormais eux seuls se chargeaient du rapport à remettre aux pouvoirs centraux en vue d'officialiser la prise de fonction des consuls (Ministerio de Estado 1887).

6 AMAE, 335CCC/12: 16/10/1874, fol. 405v.

7 AMAE, 335CCC/14: 13/12/1888, fol.241r. 
Anglais au Cap Juby, pria René Chassériau de l'accompagner à La Orotava, où se trouvait ledit chef militaire ${ }^{8}$.

Il arrivait que les bâtiments de l'État français soient affrétés pour des missions scientifiques. Entre le 28 juin et le 5 juillet 1883, durant l'escale du navire de guerre Talisman en partance pour la mer des Sargasses, la commission scientifique qui voyageait à son bord, et qui était présidée par le zoologiste Alphonse Milne-Edwards (1835-1900), reçut - lit-on dans une dépêche consulaire - la meilleure des hospitalités de la part des autorités et des habitants de l'île de Tenerife ${ }^{9}$. La première tâche du consul, dès l'arrivée du Talisman, avait été de mettre ces scientifiques «en relation avec les personnes du pays en l'état de leur être de quelque utilité ${ }^{10}$. Cette mise en relation passait précisément par l'autorité locale, laquelle fournissait généralement quelques lettres de recommandation à l'arrivant, spécialement quand celui-ci prévoyait un long séjour, mais aussi parfois par une visite aux représentants des puissances étrangères. La correspondance privée de l'anthropologue René Verneau avec Armand de Quatrefages décrit assez précisément ces étapes de la médiation préalables à l'initiation de travaux scientifiques, qui permettaient à l'arrivant de connaître une première forme d'intégration dans la société locale, avant que soit entamé un processus de sociabilisation plus ample. Le 3 juin 1877, alors qu'il venait tout juste de débarquer à Santa Cruz de Tenerife pour réaliser, avec l'appui du ministère français de l'Instruction publique, sa première mission dans l'archipel, l'anthropologue fit savoir à son collègue parisien:

Le consul de France m'a reçu avec beaucoup d'empressement; il m’a présenté aux représentants des puissances européennes et aux autorités espagnoles. Son excellence, Monsieur le Capitaine général, gouverneur de toutes les îles, m’a témoigné beaucoup de sympathies. Il m’a même rendu la visite que j'avais eu l'honneur de lui faire, ce qui est tout à fait en dehors de ses habitudes. C'est assez vous dire que je puis espérer ne rencontrer aucune entrave de la part des autorités. Bien plus, je suis assuré de leur concours ${ }^{11}$.

On pourrait multiplier encore les témoignages de bienveillance de la part des autorités locales envers la France sur laquelle insiste la correspondance consulaire, mais l'on retiendra ici, non seulement le caractère très ritualisé des visites à l'autorité grâce auxquelles, malgré le cérémonial qui encadrait les échanges, s'établissait une première forme de relation tout en fixant chacun dans une position particulière, mais aussi le rôle de médiateur que jouaient les consuls en instaurant le contact entre l'arrivant et les autorités locales, ce qui,

8 AMAE, 15CPC/96: le contre-amiral Ribell au ministre de la Marine et des Colonies, Dakar, 28/09/1886, fol. 298r-298v.

9 AMAE, 335CCC/13: 7/07/1883, fol. 185v-186r.

10 AMAE, 335CCC/13: 7/07/1883, fol. 185v.

11 BMNHN, Ms.2254: R. Verneau à A. de Quatrefages, Tenerife, 3/06/1877, fol. 346r-346v. 
dans un second temps, facilitait des relations de plus grande envergure avec la population.

\section{Les événements ponctuels à caractère festif}

Le stationnement d'escadres espagnoles ou étrangères dans la rade de Santa Cruz de Tenerife occasionnait la présence de nombreux étrangers dans la ville, et n'engendrait pas uniquement des visites de courtoisie aux autorités. Bien au contraire, la presse en parle comme de véritables événements qui touchaient l'ensemble de la population locale et déclenchaient des festivités. Lorsque ces bâtiments étaient français, le consul de France était parfois amené à organiser de fastueuses réceptions au consulat (buffets, parfois un bal ou un thé dansant [Zerolo 23/11/1881: 352; La Opinión 01/02/1886:2,30/05/1888:3]), ce à quoi la ville pouvait répondre par des réjouissances diverses, notamment par un bal d'étiquette dans les salons du Casino, le cercle le plus ancien de la ville (Zerolo 08/12/1881: 367). Ces moments venaient rompre la rigidité et l'exiguïté du cadre dans lequel les premiers échanges officiels avaient eu lieu (les visites de courtoisie, dans un univers exclusivement masculin) en rendant possible un brassage social et culturel, des rapports plus diversifiés entre un plus grand nombre de personnes: représentants de l'autorité, officiers de tous les grades, population civile; jeunes et moins jeunes; Espagnols et Français; hommes et femmes, etc.)

C'est dans la presse que nous trouvons le plus de références à ces pratiques marquées par la mixité culturelle. Dans son numéro du 23 novembre 1881, la Revista de Canarias fit la chronique d'une réception donnée par René Chassériau quelques jours plus tôt en l'honneur des aspirants du croiseur-école Flore et de l'escadre d'instruction composée des navires Résolue, Favorite et Alceste, placée sous le commandement de l'amiral Perier de Hauterive, qui stationnaient dans la rade de Santa Cruz. La revue, sous la plume de son directeur Elías Zerolo, rapporta que le consul avait voulu, par ce rassemblement, que «la distinguida oficialidad y los jóvenes aspirantes conocieran a nuestras bellas paisanas, reuniéndolas a este fin en sus elegantes salones» (Zerolo 23/11/1881: 352). À première vue, cette réception était ouverte à une population locale assez diversifiée, incluant officiers, consuls et population civile - y compris féminine -, et, bien évidemment, appartenant à un milieu social aisé. Cependant, la description n'est pas suffisamment détaillée pour que nous puissions en connaître très précisément la composition, et l'on ignore selon quels critères avaient été adressées les invitations. Était-ce vraiment la bonne société dans son ensemble qui était concernée par ces rencontres ou bien étaient-ce les pouvoirs politiques, économiques et intellectuels de la ville qu'on voulait y voir «représentés »?

D’autres réceptions données au consulat de France, comme celle qui eut lieu quelques mois plus tôt, le 8 janvier 1880, en réponse à la cérémonie publique, mais non officielle, de remerciements à la France organisée sur l'initiative de la presse sous la devise « ¡Viva España! ;Viva Francia! „Viva la Fraternidad! », pour 
les secours apportés par le pays aux victimes des inondations de Murcie en 1879 (Zerolo 08/01/1880: 16, 23/01/1880: 30-31), révèlent que les participants à ces manifestations incluaient la «colonie» française (des familles de commerçants et négociants pour la plupart), les autorités, les fonctionnaires, le corps consulaire, les sociétés et les cercles de la ville, ainsi que la presse ${ }^{12}$. Ce classement des participants - sans noms ni prénoms - en différentes catégories révèle que cette sociabilité mondaine reproduisait les structures de la société locale - les élites aux commandes de la vie politique, économique et culturelle de la capitale provinciale. Ces pratiques mettaient donc en scène des collectivités de la ville, lesquelles étaient parfois représentées par un seul de leurs membres (le capitaine général, le gouverneur civil, el alcalde, le doyen des consuls, les présidents des sociétés et des cercles, les directeurs de journaux, voire le doyen de la presse), qui pouvait se rendre à ces manifestations, suivant les circonstances, accompagné de sa famille.

Entre les visites très encadrées par le protocole et cette sociabilité mondaine, il existait assurément d'autres pratiques intermédiaires. La correspondance consulaire montre que René Chassériau ne rechignait jamais à remercier les autorités locales pour leur collaboration au bon déroulement des missions scientifiques françaises dans l'île de Tenerife, en créant des rencontres conviviales dans un cadre moins ouvert que celui des grandes réceptions. Pour donner un exemple, en mai 1885, à l'issue du séjour de l'hydrographe Anatole Bouquet de La Grye, chargé par l'État français de mesurer les longitudes entre le Sénégal, Tenerife et Cadix, le consul prit l'initiative d'organiser un dîner de remerciement à l'attention des autorités de la ville, des généraux et des colonels d'artillerie, qui avaient mis à disposition de l'ingénieur, pour la bonne exécution de ses travaux, des sous-officiers, des soldats du génie, ainsi que le fort de San Pedro' ${ }^{13}$.

Enfin, il est à noter que, en plus d'être organisateur de réceptions dès que se présentait l'occasion de mettre la France au premier plan, le consul de France prenait part aux manifestations et aux cérémonies les plus diverses, graves ou festives, qui venaient rythmer la vie dans le port à chaque fois que le protocole ou la convenance l'exigeait. Il pouvait s'agir de funérailles ou de l'exhumation d'officiers étrangers décédés à Santa $\mathrm{Cruz}^{14}$, ou encore de dîners d'adieu solennels tels que celui organisé pour le départ du capitaine général Valeriano Weyler (Maffiotte La Roche 15/11/1883: 71-72), qui coïncida avec l'inauguration, le 6

12 AMAE, 335CCC/13: 10/01/1880, fol. 111r-114v.

13 AMAE, 335CCC/14: 25/05/1885, fol. 9r-12v.

14 La cérémonie d'exhumation, en décembre 1886, du corps de l'amiral Patricio Lynch Zaldivar, ministre plénipotentiaire du Chili en Espagne, décédé sur les côtes de Tenerife en mai de la même année, fut présidée par le capitaine général, assisté de tout le corps consulaire. En tant que doyen des consuls à cette époque, René Chassériau avait occupé une place d'honneur par rapport aux autres: pendant le transport du cercueil jusque dans le Blanco Encalada, navire qui devait transporter le défunt ministre au Chili, il avait tenu l'un des cordons du poêle (AMAE, 335CCC/14: 10/12/1886, fol.119r-122v; El Seminario 12/12/1886: 180). 
décembre 1883, du câble télégraphique reliant Tenerife à Cadix (La Ilustración de Canarias 1-30/12/1883).

\section{Les fêtes régulières}

La presse canarienne signale la présence des consuls, parfois représentés par leur doyen, aux événements solennels organisés annuellement dans le cadre des principales fêtes dont la capitale était le théâtre, comme les Fiestas de Mayo et la commémoration de la victoire espagnole sur l'amiral Nelson en juillet 1797. Pendant toute la décennie de 1880, et surtout à partir de 1890, il est arrivé que les principales associations de la ville fassent coïncider certains événements relevant de leurs activités propres avec ces grands moments. Ce fut le cas de la société patriotique Sociedad Económica de Amigos del País de Santa Cruz de Tenerife (SEAPSC), qui planifia sa remise de prix annuelle lors des festivités de juillet 1880 et son exposition d'art et d'agriculture avec celles de mai 1892, ce qui donnait l'occasion d'inviter là encore les autorités, les corporations, notamment le corps consulaire, les présidents des autres sociétés et les directeurs des journaux de la capitale (El progreso de Canarias 27/07/1880: 1; Diario de Tenerife 23/04/1892:2-3, 30/04/1892: 2-3; La Opinión, 5/05/1892: 1-3). De son côté, le Gabinete Instructivo, l'une des principales associations culturelles de la ville, profitait de ces grandes dates pour organiser des veillées littéraires, et la Sociedad Filarmónica Santa Cecilia, pour donner des concerts. Les célébrations à caractère religieux faisaient également partie des grands moments de rassemblement. De même qu'ils avaient le devoir d'être présents pendant les solennités publiques, de même les consuls français avaient l'obligation d'assister à ces manifestations religieuses (Clercq et Vallat 1858: 122). À ces occasions, le corps consulaire dans son ensemble était au premier plan, se tenant aux côtés des notabilités, comme on le constate dans la presse postérieure à $1890^{15}$.

En réalité, le calendrier des consuls était également ponctué d'événements réguliers décidés depuis l'extérieur. La représentation de la France aux îles Canaries célébrait la fête nationale du 14 juillet, ce qui donnait l'occasion de placer sa demeure officielle au premier plan de la capitale, et d'y accueillir les amis du pays. Organisée pour la première fois par René Chassériau en 1881, cette célébration n'était pas le fruit d'une initiative personnelle, mais répondait à des directives ministérielles précises qui avaient été communiquées aux agents français à l'étranger par la circulaire du 26 juillet 1880, quelques semaines après le vote parlementaire établissant l'adoption du 14 juillet comme fête nationale annuelle (loi du 6 juillet 1880). Le devoir des représentants de la République

15 Au cours des fêtes de mai 1891, on put voir assister l'ensemble du corps consulaire à la misa de campaña qui commémorait l'anniversaire du début de la conquête de Tenerife en 1494 (Diario de Tenerife 30/04/1891: 2), et les deux années suivantes, on le vit réuni dans un cortège qui partait de la casas consistoriales jusqu'à l'Église paroissiale Matriz, puis, une fois les offices terminés, retournait au lieu de départ, portant avec lui l'étendard de ville (Diario de Tenerife 23/04/1892:3, 20/04/1893:3). 
à l'étranger - soulignait le texte - était «d'affirmer par une manifestation extérieure leur solidarité avec les sentiments qui animent la France entière, sans toutefois entrer en contradiction avec les usages du pays de leur résidence» ( $L M D$ 1880: 554), ce qui pourrait se traduire par l'illumination du consulat, l'exhibition du drapeau et des couleurs françaises aux fenêtres.

Même si on recommandait une attitude de bienveillance à l'égard des nationaux qui souhaiteraient donner un certain éclat à l'événement, aucune consigne particulière n'était donnée, ni dans cette circulaire ni dans la suivante, datée d'avril 1881 ( $L M D$ 1881:276), quant à d'éventuelles réceptions (dîners, buffets, bals) à organiser pour l'occasion. Les somptueuses soirées qui furent données certaines années au consulat de France (en 1881 et 1883) semblent émaner d'initiatives prises localement, par le consul en personne, vraisemblablement après en avoir donné l'avis à l'autorité locale (Clercq 1898: 135). Pendant ces soirées, dont le nombre d'invités fut variable chaque année, se retrouvaient de nouveau les ressortissants français, les autorités civiles et militaires, le corps consulaire, les présidents des principales sociétés, la presse, mais aussi les familles de ces personnalités. Dès les jours suivants, la presse s'empressait de se faire l'écho de ces moments partagés, vantant l'hospitalité reçue dans un langage qui répercutait tout l'art du paraître et du savoir-être, les «ricos vinos» et "delicadas pastasy sorbetes» qu'on avait pu déguster (Zerolo 23/07/1881: 223-224), la satisfaction (et autosatisfaction) générale, les sentiments amicaux du pays envers les représentants de la France, et ses organes républicains se félicitaient que l'on célèbre à Santa Cruz «el hecho glorioso de la toma de la Bastilla» (La Democracia 12/07/1882: 2). Mais il arriva aussi que René Chassériau limite le nombre de ses invités et que ces rencontres en dehors du cadre strict de la représentation solennelle se produisent portes fermées sur l'extérieur, à huis clos, ce dont la presse rendit compte en signalant que les personnes conviées l'avaient été en leur qualité de "amigos» du consul (Diario de Tenerife 15/07/1889: 1-2), et non en celle de représentants d'une autorité ou d'une corporation quelconque, ce qui faisait glisser la rencontre vers la sphère privée et la relation personnalisée, un point sur lequel nous allons revenir.

Pour résumer, ce panorama a fait apparaître une représentation consulaire multi-dimensionnelle, dont on a relevé deux grandes tendances. La première, politique et fortement ritualisée, dans un environnement masculin, enfermait le consul dans des cérémonies et des relations encadrées par le protocole (visites à l'autorité, solennités publiques, cérémonies religieuses, etc.), et ne laissait aucune place à l'expression des individualités. La seconde, mondaine, parfois en aval de la première, se déployait lors de festivités ou de réceptions auxquelles participait la société locale, et permettait au consul d'affirmer son ancrage dans la ville. Enfin, à l'interface de ces deux ensembles se déroulaient des pratiques de sociabilité et de représentation intermédiaires que nous allons tâcher de mieux définir. 


\section{Le consulat dans la ville: espace de la représentation, espace de la sociabilité mondaine ou espace privé?}

Afin de comprendre les enjeux de la représentation consulaire, les espaces de la mise en scène doivent être analysés sur plusieurs niveaux. En effet, les pratiques de sociabilité inhérentes à la représentation se produisaient non seulement à des moments précis, mais également dans des lieux concrets, soit à l'intérieur des consulats, soit à l'extérieur, dans des endroits clefs du centre de la ville. Aussi, il convient d'examiner l'emplacement du consulat de France dans l'espace urbain, les lieux où se déroulaient les visites et les rassemblements collectifs, ainsi que le consulat comme espace de vie.

L'occupation de l'espace urbain met tout particulièrement en valeur la recherche de visibilité des représentations étrangères et le caractère mondain de la sociabilité des consuls. Il est intéressant d'observer que, en octobre 1883, par rapport à la configuration de la ville de Santa Cruz de Tenerife, dont le centre s'était développé autour de la plaza de la Constitución, une partie des consulats, dont ceux de la Belgique, des Pays-Bas, de la France et des États-Unis d'Amérique, était installée dans la très centrique rue de la Marina, à proximité du port, des hôtels, des maisons de commerce, des magasins et de la promenade Alameda del muelle (La Ilustración de Canarias 15/10/1883: 52). D'autres étaient établis dans la rue Castillo ou dans les ruelles adjacentes (Diario de Tenerife 23/03/1886: 3). Il en était de même pour le siège des autorités, des différentes corporations, sociétés et cercles de la ville. L'ancien couvent franciscain de San Pedro de Alcántara regroupait les casas consistoriales (la mairie), la Diputación provincial, le tribunal de première instance, ainsi que le Gouvernement civil, avant que celui-ci ne soit transféré, en 1890, au Palacio de Carta, donnant directement sur ladite place (Cioranescu 1978: 70-76; 151). Seul le palais de la capitainerie générale se trouvait un peu à l'écart depuis que son siège avait été transféré, en 1882, dans le nouveau quartier en construction près de l'actuelle plaza de Weyler'16 (Díaz 1991: 563).

C'est à l'intérieur de ce court périmètre que le consul jouait son rôle de représentation et que se déroulait l'essentiel de ses pratiques de sociabilité. Certains événements festifs (concerts, défilés, etc.) et les cortèges funéraires avaient lieu dans un environnement extérieur, selon un parcours précis dans la ville. Les autres se déroulaient à huis clos, dans des espaces reflétant l'aisance, décrits par la presse comme richement décorés et rayonnant de lumière: au Palais de la Alameda de Weyler, «iluminado y exornado con gusto " (Maffiotte La Roche 15/11/1883: 71), «en los elegantes salones» du Casino (Zerolo 08/12/1881 : 367), ainsi que dans les «suntuosos salones» du consulat de France

16 Pour une description plus détaillée du centre de la capitale qui tient compte de la façon dont y ont été intégrés les espaces de sociabilité au cours du XIX ${ }^{\mathrm{e}}$ siècle, voir la thèse de doctorat de Víctor Alonso Delgado (2015: 44-63). 
(Maffiotte La Roche 31/10/1882: 68), «radiantes de luz y decorados con tanto gusto como riqueza artística» (Zerolo 23/11/1881:352).

Ce discours journalistique sur la représentation décrit les espaces de rencontres comme des salles de spectacles, et non comme des lieux de vie ou des lieux où s'exerçaient des professions spécifiques. Il met également en scène un jeu d'acteurs, d'individus presque irréels ou déconnectés du réel, et dépeint la ville de Santa Cruz, à ces occasions-là, comme un grand théâtre urbain. Tout comme le positionnement des consulats dans la ville avait un sens, la place assignée aux consuls lors des rencontres avec leurs homologues, les autorités et les autres notabilités de la capitale était très codifiée. Cette prise de possession symbolique de l'espace qui marquait les hiérarchies, passait tout d'abord par le costume: les visites à l'autorité, aux officiers haut gradés, ainsi que les cérémonies officielles et toutes autres manifestations solennelles se faisaient en uniforme (Clercq 1898: 123-124). Ainsi, lors d'un banquet organisé au consulat de France à l'occasion du passage d'une escadre française - rapporta la presse -, on avait vu se côtoyer «el uniforme de la marina francesa, el de los cónsules de otras naciones, los de diferentes institutos del ejército español, y el anti-estético frac» (Zerolo 23/11/1881: 352) - porté par les civils -, ainsi que des «elegantes señoritas» (Zerolo 23/11/1881: 352). Sans entrer dans une étude linguistique et stylistique détaillée de ce type de discours, on constate que tant l'ordre d'énumération des participants que les métonymies et les adjectifs utilisés pour qualifier les populations civiles, notamment les femmes, dont la fonction sociale est réduite à celle du simple décor, rendent compte de l'organisation symbolique de l'espace social. La place de chacun, que l'on parle de l'organisation interne de cortèges ou de l'assignation de sièges en assemblées, dans des gradins, ou à table, pour des dîners, était également déterminée par des règles de préséance strictes (Clercq 1898: 154-155) ${ }^{17}$. Cette maîtrise des codes d'interaction faisait partie, tout comme le contrôle du pouvoir politique, économique et culturel, de ce qui définissait les élites (Lilti 2001: 105-106).

Certaines variations dans le cérémonial pouvaient toutefois se produire en fonction de l'événement célébré, mais notre propos n'est pas ici de les passer en revue. En revanche, il nous semble intéressant de revenir plus en détail sur les espaces réels, plus précisément sur le consulat de France, afin de l'examiner de l'intérieur. Qui occupait le consulat? Quelles étaient les fonctions de ce lieu? Si le bâtiment situé au 11 rue Marina fut bien l'un des lieux où se joua la représentation de la France pendant la décennie de 1880, s'il en était sa vitrine, l'enceinte en question ne regroupait pas, ou n'a pas toujours regroupé, l'ensemble du personnel du poste consulaire français de la ville. En effet, en 1886, quoique restant à proximité, au numéro 80 rue Castillo, c'est-à-dire dans le périmètre défini antérieurement, la chancellerie en était séparée. Le guide de

17 Pour des exemples de l'application de ces règles de préséance à Santa Cruz de Tenerife, voir El Progreso de Canarias (27/07/1880: 1) et Maffiotte La Roche (15/11/1883: 71-72). 
Santa Cruz de Tenerife de 1886 ne signale aucun autre poste consulaire dans cette même situation, ce qui, de ce point de vue, ferait du poste français un cas exceptionnel dans la ville (Diario de Tenerife 23/03/1886: 3). En revanche, il est à noter que, au $\mathrm{n}^{\circ} 11$ de la rue Marina, se trouvait également la résidence du vice-consulat de Russie, dirigé par Abel de Aguilar. Cette coïncidence était loin d'être le fruit du hasard. Une relation étroite fondée sur un lien familial unissait le consul et le vice-consul, à tel point qu'ils furent amenés à se remplacer mutuellement dans la gérance des affaires de France et de Russie, quand l'un ou l'autre s'absentait de l'archipel (BOC 27/05/1887: 1) ${ }^{18}$. Abel de Aguilar était le gendre de René Chassériau. En 1879, il avait épousé Louise Marie Armande, née au Blanc le 17 août $1859^{19}$, fille de ce dernier et de Marie Jeanne-Amélie Bret La-Chèze (Fernández 1859: 737).

L'image d'un consulat comme espace assigné à une famille (celle des barons Chassériau) se confirme dans la presse, laquelle témoigne de la façon dont étaient perçues les réunions de société dans l'enceinte consulaire, auxquelles elle-même participait fréquemment. Que cela soit pour les réceptions organisées en l'honneur d'officiers français ou pour la célébration de la fête nationale du 14 juillet, il n'est pas toujours clair dans les journaux si l'amphitryon était le consul, le «baron» ou la «baronne», et si l'endroit de la rencontre représentait, à ce moment-là, le territoire étranger ou bien la demeure familiale. Au contraire, on y lit, au sujet de la soirée du 23 novembre 1881 organisée en l'honneur du croiseur-école Flore et de l'escadre d'instruction sous le commandement du contre-amiral Perier de Hauterive, que les invitations avaient été adressées par «el señor cónsul y su apreciable señora (Zerolo 23/11/1881:352). De la même manière, à propos de celle qui eut lieu le 14 juillet 1881, il est précisé que «los honores de la elegante morada los hizo la distinguida esposa del señor cónsul, la señora Baronesa Chasserieu [sic]» (Zerolo 23/07/1881: 223-224), cet art de recevoir rappelant à bien des égards la sociabilité mondaine qui se déroulait dans les salons de l'aristocratie au XviıI ${ }^{e}$ siècle (Lilti 2001). Si le titre de "consul» par lequel René Chassériau est désigné correspond bien à son statut d'autorité étrangère socialement reconnue, l'allusion au couple Chassériau renvoie à une famille, et le mot «baronesa», à l'extraction sociale de l'épouse. Ainsi, le consulat apparaît à la fois comme un espace de la représentation, un espace de la sociabilité mondaine et un espace privé. Non seulement représentation et sociabilité mondaine sont parfois étroitement liées, mais en plus il est difficile de séparer le consul du consulat, et l'homme public de l'homme privé.

18 Voir aussi AMAE, 393QO/890, dépêche ministérielle à R. Chassériau (minute), 10/05/1880; ibid., R. Chassériau au ministre, Tenerife, 2/05/1882.

19 AD 36 [En ligne], État civil, naissances, Le Blanc, 1853-1863, 3E 018/33, image $307 / 485<$ http://www.archives36.fr/f/EtatCivil/tableau/?\&crit1=4\&v_4_1=Blanc+\%2 8Le\%29> (consulté: 21/06/2020). 


\section{De la relation formelle à la relation personnelle: le cas des dîners et des loisirs}

Dîners, banquets, thés dansants et bals d'étiquette en général faisaient partie des pratiques de sociabilité auxquelles les notabilités de Santa Cruz étaient ponctuellement conviées au consulat de France, et au cours desquelles celles-ci se retrouvaient, échangeaient, créaient des liens. On ne reviendra pas sur le fait que, dans certains contextes, les pratiques conviviales sont des manifestations politiques où s'exhibent des hiérarchies sociales et où l'on marque son appartenance à une collectivité (Nadeau 2009: 251-261; Stefanini 2016). Pour le consul, recevoir au consulat était une façon de mettre en valeur le prestige étatique, mais aussi de remplir une obligation morale, en rendant courtoisement aux autorités, aux corporations, à la presse et aux personnalités importantes de la capitale canarienne les invitations que celles-ci lui avaient adressées antérieurement, ou afin de les remercier pour leurs manifestations de sympathie envers le pays qu'il représentait (Castro 1886b: 239).

Toutefois, dîners et loisirs paraissent devoir être traités séparément des banquets et bals d'étiquette, où les élites locales et les officiers de passage se trouvaient réunis. La correspondance de René Chassériau aussi bien que la presse laissent entrevoir l'équivalent d'une diplomatie semi-officielle, voire privée, qui se déroulait en coulisse, et grâce à laquelle le consul renforçait des liens préexistants ou en créait de nouveaux de sorte à agrandir un réseau de contacts. On pense tout d'abord aux invitations individuelles ou en nombre limité qu'il adressait aux autorités territoriales ou à ses homologues consuls, ce qui, parfois, put permettre de temporiser des relations tendues avec certains d'entre eux. Sa relation personnelle avec le consul général de Belgique, Félix Desguin, qui faisait preuve de «mauvaises dispositions » à l'endroit de la France ${ }^{20}$ - d'après lui -, était loin d'être excellente. Néanmoins, le dîner privé, chez lui, rendit possible la communication. Aux alentours du 20 juin 1883, le Belge s'était rendu chez René Chassériau, et, quoiqu' «habile et réservé», il avait livré à son hôte un certain nombre de renseignements sur les installations belges de la côte d'Afrique $^{21}$. On pense encore au dîner donné en 1885, lors du séjour d'Anatole Bouquet de La Grye, ou aux moments partagés avec des personnalités comme le grand-duc Nicolas de Russie, en septembre 188722, et le prince Marcellin Czartorski, en mars 1889 (Diario de Tenerife 26/03/1889: 3).

Loisir et convivialité généreraient un climat propice à la confiance et au dialogue. Il est frappant que ces rencontres n'aient pas toujours eu lieu au consulat de France, mais parfois à la campagne, à Tegueste, dans la maison privée de René Chassériau. Le changement de cadre, de la capitale à la campagne,

20 AMAE, 335CCC/14: 11/04/1887, fol. 146r-146v.

21 AMAE, 335CCC/13: 02/07/1883, fol. 176r-178r.

22 AMAE, 15CPC/96, R. Chassériau au ministre des Affaires étrangères, Tenerife, 30/09/1887, fol. 312r-317v. 
faisait basculer la représentation consulaire vers une rencontre de loisir, et la relation formelle, vers une relation plus personnelle. C'est bien ainsi que la presse perçut la réunion organisée en avril 1888 dans cette propriété, c'est-à-dire comme une promenade à la campagne ayant permis une réunion entre "amis", parmi lesquels se trouvaient le gouverneur civil, le délégué aux Finances et un haut officier de la Marine espagnole:

Hace pocos días, el Sr. cónsul de Francia obsequió á varios de sus amigos con una espléndida comida en la finca que posee en Tegueste.

Entre los invitados se hallaban el Sr. Gobernador civil, Delegado de Hacienda y Comandante del Vulcano, saliendo todos muy complacidos de tan agradable expedición campestre. (Las Canarias 28/04/1888:3)

En 1894, alors qu'il avait abandonné ses fonctions consulaires depuis près de cinq ans, l'ex-consul continuait à organiser des dîners dans sa propriété rurale et s'affichait avec le capitaine général, ainsi qu'avec le gouverneur civil et sa famille (Diario de Tenerife 25/04/1894: 2), ce qui fait ressortir la porosité de la frontière entre la relation officielle, exigeant certaine distance, et la relation plus intime, qui restait possible, entre les consuls, les représentants du pouvoir et les notabilités locales de manière générale. On remarquera toutefois, d'après les témoignages que nous avons réunis, que cette sociabilité plus discrète et plus individualisée que celle des grandes réceptions, ne semble avoir engagé René Chassériau dans une relation rapprochée qu'avec les autorités politiques et militaires, ses homologues consuls, certains fonctionnaires et les grands officiers uniquement. Les directeurs de journaux et les présidents des sociétés paraissent en avoir été exclus, ce qui renvoie peut-être à l'existence de cloisonnements au sein même de l'élite de la capitale.

Pour résumer, si cette sociabilité «en petit comité» et à l'écart de la scène urbaine put permettre à chacun d'exprimer temporairement son individualité, celle qui se déroulait au sein de la capitale provinciale et qui engageait l'ensemble des institutions, des corporations et des sociétés locales ne peut être dissociée des fonctions de représentation publique remplies par les principaux membres de ces dernières. Sa dynamique interne était régie par la «réciprocité», un principe clé de la diplomaticité (Villar 2006: 97-98), puisque chacun, enfermé dans un rôle précis, y occupait la place de l'invité ou de l'organisateur, et partageait avec l'autre les moments importants qui rythmaient la vie locale. Les réunions privées au consulat paraissent avoir constitué un "entre deux», où le consul, tout en sa qualité de représentant d'un État étranger, nouait des relations courtoises, voire «amicales», plus ou moins conditionnées par ses fonctions.

\section{Conclusion}

Cette étude montre que les pratiques de sociabilité consulaire, très théâtralisées du fait des convenances mondaines, des règles de préséance et du protocole en général, ne peuvent être extraites du cadre plus large dans lequel se déroulait une sociabilité qui impliquait, au sein de la capitale provinciale des Canaries, 
les principales institutions, corporations et sociétés liées au pouvoir politique, économique, intellectuel et artistique local. Y participer était une manière, pour les consuls, non seulement de respecter le protocole consulaire et d'accentuer la visibilité du pays représenté, mais aussi de marquer un ancrage dans la ville de résidence. On notera le caractère essentiellement mondain, élitiste et cloisonné de cette sociabilité. Les somptueuses soirées organisées par l'autorité ou par René Chassériau, aussi bien que les veillées littéraires du Gabinete Instructivo ou la distribution des prix aux lauréats des concours annuels proposés par la SEAPSC, ne pouvaient s'adresser qu'à la minorité détentrice du savoir et du pouvoir de la capitale. Tout en respectant le protocole, chaque consul avait une approche différente de sa sociabilité. Si les rapports officiels avec les autorités territoriales étaient rigoureusement régulés, une sociabilité plus souple, fondée sur la convivialité et le loisir, semblait favoriser des relations plus personnelles, ce qui nous a amenée à montrer que, parfois, la frontière entre le rapport professionnel et le rapport privé était assez floue. On peut se demander dans quelle mesure la possibilité de s'entretenir de façon individuelle avec certains officiers et avec l'autorité, notamment lors de dîners ou d'autres moments de convivialité, n’a pas eu de répercussions bénéfiques sur l'accomplissement des missions consulaires, en favorisant un rapport de confiance. Enfin, il ressort que, en tant que médiateur interculturel, le consul facilitait la mise en rapport et l'échange entre, d'un côté, les ressortissants français qui séjournaient, même brièvement, dans le port (officiers, scientifiques en mission officielle) et, de l'autre, les autorités et l'élite locales.

\section{Bibliographie}

Abélès M., 1991, "Mises en scène et rituels politiques. Une approche critique", Hermès, La Revue, vol. 8-9, n 1, p. 241-259.

Agulhon M., 1977, Le cercle dans la France bourgeoise, 1810-1848. Étude d'une mutation de sociabilité, Paris, Colin.

Alonso Delgado V., 2015, Deporte, ocio y sociabilidad en las islas Canarias occidentales (1850-1936), thèse de doctorat sous la direction de Xavier Pujadas i Martí, Departament de Activitat Fisica i Ciencies de l'Esport, Université Ramón Llull, <https://www.tdx.cat/handle/10803/285238>, consulté le 04/06/2020.

Anónimo, 23/11/1886, «Guía de Santa Cruz de Tenerife, oficinas y establecimientos públicos y autoridades principales», Diario de Tenerife, p. 3.

Calvo C., 1885, Dictionnaire manuel de diplomatie et de droit international public et privé, Berlin/Paris, Puttkamer \& Mühlbrecht/Guillaumin/Pedone-Lauriel/ Rousseau.

Castro y Casaleiz A. (de), 1886a, Guía práctica del diplomático español, t. 1, Madrid, Establecimiento tipográfico El Correo.

Castro y Casaleiz A. (de), 1886b, Guía práctica del diplomático español, t. 2, Madrid, Establecimiento tipográfico El Correo. 
Chartier R., 1989, «Le monde de la représentation », Annales. Économies, Sociétés, Civilisations, $44^{\mathrm{e}}$ année, $\mathrm{n}^{\circ}$ 6, p. 1505-1520.

Cioranescu A., 1978, Historia de Santa Cruz de Tenerife, Santa Cruz de Tenerife, Confederación española de cajas de ahorros, vol.3.

Clercq A. (de) \& Vallat C. (de), 1858, Guide pratique des consulats, t. 1, 2e édition Paris, Amyot.

Clercq J. (de), 1898, Guide pratique des consulats, t. 1, 5e édition, Paris, Pédone.

De Goey F., 2016, "Les consuls et les relations internationales au XIX ${ }^{e}$ siècle», Cahiers de la Méditerranée, vol. 93, p. 61-75, <http://journals.openedition.org/ cdlm/8492>, consulté le 26/12/2019.

Díaz Pérez A.M., 1991, «Las distintas sedes de la capitanía general de Canarias en Santa Cruz de Tenerife», VIII Coloquio de Historia Canario-Américana (1888), Las Palmas, vol. 2, p. 551-570.

Fernández de Bethencourt F., 1859, Nobiliario de Canarias, t. 3, La Laguna, Régulo editor.

[GDE] Guía diplomática de España, 1887, Madrid, Imprenta M. Tello.

Kingston de Leusse M., 1998, Diplomate. Une sociologie des ambassadeurs, Paris, L'Harmattan.

Leuwers H., 2005, «Pratiques, réseaux et espaces de sociabilité au temps de la Révolution française», in Martin J.-C. (coord.), La Révolution à l'œuvre: perspectives actuelles dans l'histoire de la Révolution française, Rennes, Presses universitaires de Rennes, <https://books.openedition.org/pur/16026?lang=fr>, consulté le 30/04/2020.

[LMD] Simon E. (coord.), 1880-1881, Le Memorial diplomatique, Paris, Kugelmann.

Lilti A., 2001, «Sociabilité mondaine, sociabilité des élites? Les salons parisiens dans la seconde moitié du XviII ${ }^{\mathrm{e}}$ siècle», Hypothèses, vol. 4, nº 1, p. 99-107.

Maffiotte La Roche L. [pseud. Ortiguilla], 31/10/1882, «La quincena [Rubrique]», La ilustración de Canarias, p. 68.

Maffiotte La Roche L. [pseud. Ortiguilla], 15/11/1883, «La quincena [Rubrique]», La ilustración de Canarias, p. 71-72.

Ministerio de Estado, 1887, Reglamento para la admisión de los cónsules, vicecónsules y agentes consulares extranjeros en España y en sus provincias $y$ posesiones de Ultramar, Madrid, M. Minuesa de los Ríos.

Nadeau R., 2009, «Le banquet, intégration et sociabilité citoyenne dans la cité grecque », Hypothèses, vol. 12, $\mathrm{n}^{\circ} 1$, p. 251-261.

Poggi Borsotto F.M., 1881, Guía histórica descriptiva de Santa Cruz de Tenerife, Santa Cruz de Tenerife, Imprenta isleña.

Sintomer Y., 2013, «Les sens de la représentation politique: usages et mésusages d'une notion", Raisons politiques, vol. 2, n 50, p. 13-34.

Stefanini L. (dir.), 2016, À la table des diplomates. L'histoire de France racontée à travers ses grands repas (1520-2015), Paris, Liconoclaste/Gallimard. 
Villar C., 2006, Le discours diplomatique, Paris, L'Harmattan.

Zerolo E. [pseud. L. Rio Oseleza], 08/01/1880, «Conversación quincenal [Rubrique]», Revista de Canarias, p. 16.

Zerolo E. [pseud. L. Rio Oseleza], 23/01/1880, «Conversación quincenal [Rubrique]», Revista de Canarias, p. 30-31.

Zerolo E. [pseud. L. Rio Oseleza], 23/07/1881, «Conversación quincenal [Rubrique]», Revista de Canarias, p. 223-224.

Zerolo E. [pseud. L. Rio Oseleza], 23/11/1881, «Conversación quincenal [Rubrique]», Revista de Canarias, p. 352.

Zerolo E. [pseud. L. Rio Oseleza], 08/12/1881, «Conversación quincenal [Rubrique]», Revista de Canarias, p. 367.

\section{Sources manuscrites}

AD 36 [Archives départementales de l'Indre] [En ligne], État civil, naissances, Le Blanc, 1853-1863, 3E 018/33, <http://www.archives36.fr/f/EtatCivil/tableau/? \&crit1=3\&v_3_1=Naissances $>$, consulté le 30/11/2020.

AMAE, 335CCC/12 à 14 [Archives du ministère des Affaires étrangères - La Courneuve, Correspondance consulaire et commerciale, Sainte-Croix de Ténériffe, t. 12 (1852-1874); t. 13 (1875-1884); t. 14 (1885-1894)].

AMAE, 15CPC/96 [Archives du ministère des Affaires étrangères - La Courneuve, Correspondance politique des consuls, Espagne, Sainte-Croix de Ténériffe, $\mathrm{n}^{\circ}$ 96, 1886-1887].

AMAE, 393QO/890 [Archives du ministère des Affaires étrangères - La Courneuve, Personnel, Dossiers nominatifs, $1^{\text {re }}$ série, $n^{\circ} 890$ : René Chassériau]

BMNHN, Ms. 2254 [Bibliothèque centrale du Muséum national d'histoire naturelle - Paris, Correspondance de E. T.Hamy, vol. 1, René Verneau, 1877, fol. 346-383]

\section{Publications périodiques (années consultées)}

[BOC] Boletín Oficial de la Provincia de Canarias, 1874-1889, Santa Cruz de Tenerife.

Diario de Tenerife, 1886-1894, Santa Cruz de Tenerife.

El Seminario, 1886, Santa Cruz de Tenerife.

La Democracia, 1882, Santa Cruz de Tenerife.

La Ilustración de Canarias, 1883-1884, Santa Cruz de Tenerife.

La Opinión, 1886-1892, Santa Cruz de Tenerife.

Las Canarias, 1888, Santa Cruz de Tenerife.

Revista de Canarias, 1879-1881, Santa Cruz de Tenerife. 